\title{
ON EQUIDISTANT CUBIC SPLINE INTERPOLATION
}

\author{
BY I. J. SCHOENBERG
}

\section{Communicated by Fred Brauer, May 14, 1971}

Let $n$ be a natural number and let $S[0, n]$ denote the class of cubic spline functions $S(x)$ defined in the interval $[0, n]$ and having the points $1, \cdots, n-1$ as knots. This means that the restriction of $S(x)$ to the inverval $(\nu, \nu+1)(\nu=0, \cdots, n-1)$ is a cubic polynomial, and that $S(x) \in C^{2}[0, n]$. The most general element of $S[0, n]$ is evidently of the form

$$
S(x)=\sum_{0}^{3} a_{\nu} x^{\nu}+\sum_{1}^{n-1} c_{\nu}(x-\nu)_{+}^{8},
$$

and is seen to depend linearly on $n+3$ parameters. Here we have used the function $x_{+}=\max (0, x)$.

The interpolatory properties of the elements of the class $S[0, n]$ have recently attracted considerable attention. The two main kinds of this so-called cubic spline interpolation are as follows.

1. Natural cubic spline interpolation. We are required to find $S(x) \in S[0, n]$ such as to satisfy the conditions

$$
\begin{aligned}
S(\nu) & =f(\nu) \quad(\nu=0, \cdots, n), \\
S^{\prime \prime}(0) & =S^{\prime \prime}(n)=0 .
\end{aligned}
$$

2. Complete cubic spline interpolation. Here we are asked to find $\tilde{S}(x) \in S[0, n]$ so as to satisfy the conditions

$$
\begin{aligned}
\tilde{S}(\nu) & =f(\nu) \quad(\nu=0, \cdots, n), \\
\tilde{S}^{\prime}(0) & =f^{\prime}(0), \quad \tilde{S}^{\prime}(n)=f^{\prime}(n) .
\end{aligned}
$$

The existence and unicity of the solutions of these problems is widely known. See [1], [2], or [5, Chapter 4] for illuminating discussions of these problems.

We may describe the solution of the first problem (2), (3) by the interpolation formula

$$
f(x)=\sum_{0}^{n} f(\nu) L_{\nu}(x)+R,
$$

where the fundamental functions $L_{\nu}(x)$ are elements of $S[0, n]$ and

AMS 1970 subject classifications. Primary 41A15.

Copyright (1) American Mathematical Society 1971 
are characterized by the conditions

$$
L_{\nu}(j)=\delta_{\nu, j} \quad \text { and } \quad L_{\nu}^{\prime \prime}(0)=L_{\nu}^{\prime \prime}(n)=0 \quad \text { for all } \nu .
$$

Likewise the solution of the second problem (4), (5) is described by the formula

$$
f(x)=\sum_{0}^{n} f(\nu) \tilde{L}_{\nu}(x)+f^{\prime}(0) \Lambda(x)-f^{\prime}(n) \Lambda(n-x)+R,
$$

where the fundamental functions $\tilde{L}_{\nu}(x)$ and $\Lambda(x)$ are elements of $S[0, n]$ and are characterized by the conditions

$$
\tilde{L}_{\nu}(j)=\delta_{\nu, j}, \quad \tilde{L}_{\nu}^{\prime}(0)=\tilde{L}_{\nu}^{\prime}(n)=0 \quad \text { for all } \nu,
$$

and

$$
\Lambda(j)=0 \quad \text { for all } j, \quad \Lambda^{\prime}(0)=1, \quad \Lambda^{\prime}(n)=0 .
$$

Until now, the solution of these problems, i.e. the construction of the interpolating spline function, has usually required the inversion of a matrix (essentially a tridiagonal matrix) of a size that was roughly $n \times n$. It is the purpose of this note to describe explicit solutions of these problems that do not require any matrix inversion.

Let $M_{1}(x)=1$ if $-\frac{1}{2} \leqq x \leqq \frac{1}{2}$, and $M_{1}(x)=0$ otherwise. The 4-fold convolution $M(x)=M_{4}(x)=M_{1} * M_{1} * M_{1} * M_{1}(x)$ is a bell-shaped frequency function with its support in the interval $(-2,+2)$. Explicitly

$$
\begin{aligned}
M(x) & =M_{4}(x) \\
& =\frac{1}{6}\left\{(x+2)_{+}^{3}-4(x+1)_{+}^{3}+6 x_{+}^{3}-4(x-1)_{+}^{3}+(x-2)_{+}^{3}\right\},
\end{aligned}
$$

an expression which shows that $M(x)$ is a cubic spline function defined for all real $x$ and having knots at the five points $-2,-1,0,1,2$. See $[6,71]$, where a graph of $M(x)$ is also given.

A relevant property of the function $M(x)$, called the cubic $B$-spline is expressed by the following:

Lemma 1. Every $S(x) \in S[0, n]$ admits a unique representation of the form

$$
S(x)=\sum_{-1}^{n+1} C_{j} M(x-j) \quad(0 \leqq x \leqq n) .
$$

Our interpolation problems are solved as soon as we determine the coefficients of the representations 


$$
\begin{aligned}
L_{\nu}(x) & =\sum_{-1}^{n+1} c_{j, \nu} M(x-j), \\
\tilde{L}_{\nu}(x) & =\sum_{-1}^{n+1} \tilde{c}_{j, \nu} M(x-j), \\
\Lambda(x) & =\sum_{-1}^{n+1} \gamma_{j} M(x-j),
\end{aligned}
$$

and therefore

$$
-\Lambda(n-x)=-\sum_{-1}^{n+1} \gamma_{n-1} M(x-j)
$$

for the last fundamental function in (8).

We need the algebraic integer

$$
\lambda=-2+\sqrt{ } 3=-.2679491924,
$$

which is the root of least absolute value of the equation $x^{2}+4 x+1=0$. Furthermore, let

$$
\lambda^{k}=a_{k}+b_{k} \sqrt{ } 3 \quad \text { for all integers } k,
$$

where $a_{k}$ and $b_{k}$ are integers. The expressions

$$
a_{k}=\frac{1}{2}\left(\lambda^{k}+\lambda^{-k}\right), \quad b_{k}=\frac{1}{2 \sqrt{ } 3}\left(\lambda^{k}-\lambda^{-k}\right)
$$

show that both sequences $\left(a_{k}\right)$ and $\left(b_{k}\right)$ satisfy the same recurrence relation

$$
x_{k+2}+4 x_{k+1}+x_{k}=0 \quad \text { for all } k,
$$

and are defined by it with the initial values

$$
a_{0}=1, \quad a_{1}=-2, \quad \text { and } \quad b_{0}=0, \quad b_{1}=1,
$$

respectively. The sequence $\left(a_{k}\right)$ is even, while the sequence $\left(b_{k}\right)$ is odd. A short table of values is as follows:

$$
\begin{array}{rrrrrrrrr}
k & -1 & 0 & 1 & 2 & 3 & 4 & 5 & 6 \\
a_{k} & -2 & 1 & -2 & 7 & -26 & 97 & -362 & 1351 \\
b_{k} & -1 & 0 & 1 & -4 & 15 & -56 & 209 & -780
\end{array}
$$

Incidentally, the numbers $x=\left|a_{k}\right|, y=\left|b_{k}\right|$ represent all integer solutions of Pell's equation $x^{2}-3 y^{2}=1$. 
Our main results are the following two theorems.

THEOREM 1. The coefficients $c_{j, y}$ of the fundamental functions (13) for natural cubic spline interpolation are described as follows:

$$
\begin{aligned}
c_{j, 0} & =2-\left(b_{n-1} / b_{n}\right) & & \text { if } j=-1, \\
& =b_{n-j} / b_{n} & & \text { if } j=0,1, \cdots, n+1 ; \\
c_{j, \nu} & =-6 b_{n-\nu} b_{j} / b_{n} & & \text { if } j \leqq \nu, 0<\nu<n, \\
& =-6 b_{n-j} b_{\nu} / b_{n} & & \text { if } \nu \leqq j, 0<\nu<n .
\end{aligned}
$$

Moreover, the symmetry relations

$$
c_{j, \nu}=c_{n-j, n-\nu}
$$

hold throughout, and in particular for $\nu=n$ we find that

$$
c_{j, n}=c_{n-j, 0}
$$

are already described by the formulae (20).

THEOREM 2. The coefficients $\tilde{c}_{j, \nu}$ and $\gamma_{j}$ of the fundamental functions (14) and (15) for complete cubic spline interpolation are as follows:

$$
\begin{aligned}
\tilde{c}_{j, 0} & =-a_{n-|j|} / b_{n}, \\
\tilde{c}_{j, \nu} & =-2 a_{j} a_{n-\nu} / b_{n} \quad \text { if } j \leqq \nu, 0<\nu<n, \\
& =-2 a_{\nu} a_{n-j} / b_{n} \quad \text { if } \nu \leqq j, 0<\nu<n .
\end{aligned}
$$

The symmetry relation

$$
\tilde{c}_{j, \nu}=\tilde{c}_{n-j, n \rightarrow \nu}
$$

holds throughout, and for $\nu=n$ we find

$$
\tilde{c}_{j, n}=\tilde{c}_{n-j, 0}
$$

already described by (24). Finally

$$
\gamma_{j}=-\frac{1}{3} a_{n-j} / b_{n} .
$$

In the application of these formulae there is never any loss of accuracy due to numerical instability, no matter how large $n$ may be. In fact, writing $c_{j, \nu}=c_{j, \nu}^{(n)}$, we have that

$$
\lim c_{j, \nu}^{(n)}=c_{j, \nu}^{+}, \quad \text { as } n \rightarrow \infty,
$$

where

$$
L_{\nu}^{+}(x)=\sum_{j=-1}^{\infty} c_{j, \nu}^{+} M(x-j) \quad(\nu=0,1,2, \cdots)
$$


are the fundamental functions of semicardinal cubic spline interpolation. Their coefficients are found to be

$$
\begin{aligned}
c_{j, 0}^{+} & =2-\lambda & & \text { if } j=-1, \\
& =\lambda^{j} & & \text { if } j \geqq 0 ; \\
c_{j, \nu}^{+} & =-6 b_{j} \lambda^{\nu} & & \text { if } j \leqq \nu, \nu>0, \\
& =-6 b_{\nu} \lambda^{j} & & \text { if } \nu \leqq j, \nu>0 .
\end{aligned}
$$

Similar results hold concerning $\tilde{c}_{j, \nu}^{(n)}$ and $\gamma_{j}^{(n)}$.

Our subject has received special attention in $[1, \S 2.4]$ and [3]. Greville's approach seems closest to ours. He determines the coefficients $a_{\nu}$ and $c_{\nu}$ of the solution $S(x)$ of (2) and (3) in its explicit form (1). In order to avoid the loss of accuracy inherent in this formulation by using integer arithmetic (as Greville recommends), one must work with a large number of digits even for moderately large values of $n$. Indeed, if $n=20$, say, products containing close to 20 digits and multiplicands of more than 10 digits appear in the calculations. The advantages of using the representation (12) in terms of $B$-splines become apparent.

Computational advice. Construct the solution $S(x)$ of the problem (2), (3) in the form (12), where the $C_{j}$ are to be determined from the relations

$$
C_{j}=\sum_{\nu=0}^{n} c_{j, v} f(\nu) \quad(j=-1,0, \cdots, n+1) .
$$

A similar remark concerns the problem (4), (5).

The results here described were obtained by means of the fundamental function and the eigensplines of cardinal spline interpolation as discussed in [7]. In the present cubic case this approach is particularly simple and intuitive. An alternative and shorter way of obtaining Theorems 1 and 2 is to use the results of D. Kershaw as described in [4]. The connection is as follows: If we introduce the representation (12) in to the equations (4) and (5), we obtain a system of $n+3$ equations for the $n+3$ unknown coefficients $C_{j}$. The matrix of this linear system is a special case of one of the matrices that were explicitly inverted by Kershaw in [4]. Similarly, the problem (2) and (3) leads to a matrix that can easily be reduced to a special case of another matrix already inverted by Kershaw. Kershaw does not point out this application of his results, an application that becomes strikingly apparent if we use the representation (12) in terms of $B$ splines. However, our approach via cardinal spline interpolation has 
the advantage of generalizing to higher degree spline interpolation problems. Indeed, in a forthcoming paper [8], written in collaboration with A. Sharma, we present the applications of our approach to quintic spline interpolation problems.

\section{REFERENCES}

1. J. H. Ahlberg, E. N. Nilson and J. L. Walsh, The theory of splines and their applications, Academic Press, New York, 1967. MR 39 \#684.

2. T. N. E. Greville, Spline functions, interpolation, and numerical quadrature, Mathematical Methods for Digital Computers, vol. II, Wiley, New York, 1967, Chap. 8, pp. 156-168.

3. - Table for third-degree spline interpolation with equally spaced arguments, Math. Comp. 24 (1970), 179-183. MR 41 \#2885.

4. D. Kershaw, The explicit inverses of two commonly occurring matrices, Math. Comp. 23 (1969), 189-191. MR 38 \#6754.

5. T. J. Rivlin, An introduction to the approximation of functions, Blaisdell, Waltham, Mass., 1969. MR 40 \#3126.

6. I. J. Schoenberg, Contributions to the problem of approximation of equidistant data by analytic functions, Parts A and B, Quart. Appl. Math. 4(1946), 45-99; 112141. MR 7, 487; MR 8, 55.

7. - Cardinal interpolation and spline functions. II. Interpolation of data of power growth, MRC Technical Summer Report \#1104, Madison, Wis., 1970; also: J. Approximation Theory (to appear).

8. I. J. Schoenberg and A. Sharma, The interpolatory background of the EulerMaclaurin quadrature formula, Bull. Amer. Math. Soc. 77 (1971), 1034-1038.

University of Wisconsin, Madison, Wisconsin 53706 